\title{
Non-communicable diseases awareness and control in a rural population in an epidemiologically advanced stage of transition (Kerala): results of the epidemiology of non-communicable diseases in rural areas study
}

\author{
Jaideep C. Menon ${ }^{1 *}$, Aswathy Sreedevi ${ }^{2}$, Beena K. V. ${ }^{3}$, Rajesh T. ${ }^{4}$, Thankachan V. Attacheril ${ }^{5}$, \\ Jaisoorya T. S. ${ }^{6}$, Vijayakumar N. ${ }^{7}$, Joseph K. Joseph ${ }^{8}$, Narayanan M. Menon ${ }^{9}$, Jacob Joseph ${ }^{10}$, \\ Ajit Thachil $^{10}$, Rakesh P. Susheelan ${ }^{2}$, Amitava Banerjee ${ }^{11}$
}

\author{
${ }^{1}$ Department of Preventive Cardiology, ${ }^{2}$ Department of Community Medicine, ${ }^{3}$ Department of Public health, \\ ${ }^{4}$ Department of Cardiology, Amrita Institute of Medical Sciences, Amrita University, Kochi, Kerala, India \\ ${ }^{5}$ Department of Cardiology, Lourdes Hospital, Kochi, Kerala, India \\ ${ }^{6}$ Department of Psychiatry, NIMHANS, Bangalore, Karnataka, India \\ ${ }^{7}$ Officer I/C, Blood Bank and Dialysis Unit, District Hospital, Aluva, Kochi, Kerala, India \\ ${ }^{8}$ Department of Nephrology, Little Flower Hospital, Angamaly \\ ${ }^{9}$ General Practitioner, Sreemoolanagaram, Aluva, Kerala, India \\ ${ }^{10}$ Department of Cardiology, Lisie Hospital, Kerala, India \\ ${ }^{11}$ Department of Cardiology and Bio-Informatics, University College of London, London, United Kingdom
}

Received: 29 March 2020

Revised: 03 June 2020

Accepted: 04 June 2020

\section{*Correspondence:}

Dr. Jaideep C. Menon,

E-mail: menon7jc@gmail.com

Copyright: (C) the author(s), publisher and licensee Medip Academy. This is an open-access article distributed under the terms of the Creative Commons Attribution Non-Commercial License, which permits unrestricted non-commercial use, distribution, and reproduction in any medium, provided the original work is properly cited.

\begin{abstract}
Background: India carries the greatest burden of non-communicable diseases (NCDs) amongst nations. It is estimated that by the year 2030 there will be 101 million diabetics, 218 million hypertensives in India. NCDs would account for $53 \%$ of deaths in India by the year 2020 of which CVD would be the cause in $43 \%$. Kerala is the diabetic and cardiovascular disease capital of India, with rates of prevalence as high as twice the national average. The ageadjusted CAD mortality rates per 100,000 is 382 for men and 128 for women in Kerala.

Methods: The study was carried out in 5 contiguous panchayats of Ernakulam district, Kerala state. The study was carried out in 2 Phases. In the first phase individuals self-reported non communicable diseases and risk factors through the medium of an ASHA administered Questionnaire. In the second phase blood tests were done in individuals from the study population in those above the age of 30 years.

Results: Of the 114,064 surveyed 63,051 individuals were above 30 years of age with a prevalence of diabetes at $11.1 \%$, hypertension $15.6 \%$ and dyslipidemia $6.9 \%$ when self-reported. In the Phase 2 of the study blood sugar, total cholesterol and blood pressure was measured in close to 8000 individuals in whom $48 \%$ of individuals with high sugars, $37 \%$ with high blood pressure and $85 \%$ with high cholesterol values were unaware of their disease status. More females were unaware of their diabetic and lipid status and males of their hypertensive status. About $25 \%$ of diabetics, $40 \%$ of hypertensives and $6 \%$ of dyslipidemics had normal values. This study provided prevalence of NCDs on a large enough scale to help in policy planning if not for the country at least for the state of Kerala.

Conclusions: The study results suggested low awareness and poor control of NCDs in the study population.
\end{abstract}

Keywords: ASHA, Diabetes, ENDIRA, Hypertension, Surveillance 


\section{INTRODUCTION}

India is arguably the Diabetic and Cardiovascular capital of the world. ${ }^{1,2}$ In India, from 2005 to 2015 , CVD, stroke and diabetes is estimated to have caused a cumulative income loss of US $\$ 233.6$ billion. ${ }^{3,4}$

Kerala is the diabetic and cardiovascular disease capital of India, with rates of prevalence as high as twice the national average being reported. The age-adjusted CAD (coronary artery disease) mortality rates per 100,000 are 382 for men and 128 for women in Kerala. The CAD rates in Kerala are higher than those of industrialized countries and 3 to 6 times higher than that of rural Japan and China. ${ }^{5-7}$

India, at present, is in the midst of a pandemic of noncommunicable or the so called lifestyle diseases. NCDs have replaced communicable diseases as the leading cause of morbidity and mortality in India. ${ }^{3,6,8}$

The reasons for this pandemic are many and include rapid urbanization, unhealthy diet habits including increased use of fast and preserved foods, lack of exercise and an increasingly sedentary life-style, increased stress levels, pollution, ageing population and widespread use of tobacco and alcohol. ${ }^{9}$ There is little contemporary data on the prevalence of CVD, diabetes mellitus, hypertension and dyslipidemia in India, at the community level. ${ }^{6-8}$ Health information gathering is an important part of any health system, but is often non-existent in low and lowmiddle income countries, or is plagued by poor quality data that is inadequate for planning health policy. Population based surveys are an invaluable source of health information. The key aim of these surveys was to provide quality data for policy development, programme planning, monitoring and evaluation. Population based surveys have been used extensively to gather information on fertility, mortality, family planning, maternal and child health, and some other aspects of health, nutrition and health care in India but as much for NCDs .

A myocardial infarction is not an uncommon occurrence in a 30 to 40 year old male in India. The mean age of occurrence of atherosclerotic CVD is 10-20 years earlier in Indians, as compared to high income countries. India is at the epicentre of this pandemic of global CVD which tends to affect a much younger subset of individuals who are usually males, most likely to be the sole bread winners of the family. ${ }^{7,8}$

It is also not uncommon to make the diagnosis of diabetes, hypertension or dyslipidemia in one who has come in with an index cardiovascular event, either a stroke or a myocardial infarction. ${ }^{10,11}$ This is so as there is no screening programme at the primary care level. The reasons for there not being a screening at the primary care level are many. $80 \%$ of outpatient and $60 \%$ of inpatient care in India is rendered through private hospitals. $30 \%$ of rural Indians still do not have access to medical facilities, nearby. $70 \%$ of health care expenditure is out of the pocket and this pushes an estimated 60 million individuals into poverty annually. ${ }^{12}$

Only about $17 \%$ of individuals in the country have some sort of an insurance cover, of which $12 \%$ is through government insurance schemes, $3 \%$ provided by employer insurance schemes and only $2 \%$ as individual self-paid insurance. ${ }^{13}$ Insurance does not cover for investigations done on an outpatient basis with most schemes only covering for inpatient treatment. Insurance premiums are also unfairly high for the high risk group of individuals on treatment for diabetes, hypertension or in those with established CVD.

All this accounts for the void in screening at the primary care level, resulting in a large number of undiagnosed cases of diabetes, hypertension and dyslipidemia, either with full-blown disease or in the pre-disease state. These are just the subsets wherein lifestyle changes, if implemented, would go a long way in preventing progression to complications like CVDs.

The ENDIRA (epidemiology of non-communicable diseases in rural areas) study gains significance given the central government's move towards a policy of universal healthcare for all and the role of the National Health Mission (NHM) in providing treatment for NCDs through health centres, all across India. ${ }^{14}$ Moreover the $\mathrm{NIH}$ sponsored, ACS-QUIK registry which was carried out in multiple centres all over Kerala has shown the scope for bringing about far reaching changes in patient management protocols through specific interventions on a pan-Kerala basis. ${ }^{15}$ It is worth pointing out that the ENDIRA study was the first in utilising front line health workers (ASHAs) for gathering data on such a large scale16. ASHAs being residents of the wards that they work in are usually aware of health conditions and other details of residents of their ward and are well placed for data collection and surveillance.

In spite of having excellent tertiary care facilities mainly in urban India, we still lack a primary or secondary preventive strategy for NCDs. This study provides prevalence of NCDs on a large enough scale to help in policy planning if not for the country at least for the state of Kerala.

The aim of the ENDIRA investigators was to estimate the prevalence of non-communicable diseases in a rural population and to estimate awareness, treatment and adequacy of control of hypertension, diabetes and dyslipidemia.

\section{METHODS}

The study was conducted in all of 75 wards of the 5 adjoining panchayats of Mookannoor (14 wards), Karukutty (16 wards), Manjapra (13 wards), Thuravoor (15wards) and Kalady (17 wards). ${ }^{16}$ The total population 
covered by the survey was 114,064 individuals of whom 63,051 individuals were over 30 years of age, our target group.

The study period was between May 2012 and 2014 for the data collection (Phase 1) and till 2016 for follow up blood investigations and physical examination in the target group (Phase 11). The study was approved by the Institutional Ethics Committee of the MAGJ Hospital Mookkannoor (Refn: IEC-MAGJ-2011-CARD-57, Dated- 21/11/ 2011).

The trial was registered with Clinical Trial Registry of India (CTRI) - CTRI/2012/01/002380. The blood tests were done in the $\mathrm{NABH}$ accredited laboratory in the General Hospital, Ernakulam.

Prior to commencement of the survey the proposal was presented and approval got, individually, from all the 5 panchayats. The questionnaire based survey was carried out by ASHA (Accredited Social Health Activist) workers after a training and orientation session for them. A kit which included a weighing machine, a measuring tape and the questionnaire booklets were handed over to each ASHA of respective wards. The standardised questionnaire having 14 questions included ones on socio-demographics, life style and medical conditions.

The study questionnaire was developed in the local language, Malayalam, based on the World Health Organization's STEPS questionnaire and modified based on inputs from ASHAs, JPHNs (Junior Public Health Nurse) and public representatives. This was then pretested in 100 individuals before printing. All the ASHAs underwent training on the administration of the questionnaire and in the measurement of height and weight. Non stretchable tapes were used for height measurement and the weighing machines were standardized before data collection. The data collected included the socio-demographic details of the family, address and socioeconomic status categorized as 'above poverty line (APL)' or 'below poverty line (BPL)' status were recorded. Anthropometric measurements such as height in $\mathrm{m}$ and weight in $\mathrm{Kg}$ of all the family members were also measured. The questionnaire also included details regarding history of diabetes, hypertension, dyslipidemia, chronic respiratory disease, surgical history, treatment for cancers, heart disease and stroke. Personal habits such as tobacco and alcohol consumption patterns, diet history of consumption of red meats, type and quantity of oil consumed, was also collected. The past surgical history, treatment for cancers, history of heart disease and stroke was also noted. The data collated was entered on a excel sheet after informed consent from each individual who was part of the survey. Each individual in the 5 panchayats was provided a unique health identification number.

Any form of tobacco use or alcohol use was considered as a behavioral risk factor for NCD. The score used was the
AUDIT (Alcohol use disorder Identification test). Tobacco dependence abuse was assessed by the Fagerstrom's test for nicotine dependence. Height and weight were measured using standard protocols.

\section{Inclusion criteria}

All individual resident of the study Panchayats who consented for the study were included in the study.

\section{Exclusion criteria}

Those unwilling to take part in the study or did not consent for the study and individuals less than 30 years of age were not included in Phase II of the study.

\section{Phase II}

In the second phase of the study the 75 wards in the 5 panchayats were divided into units of 3 wards each. One medical camp was conducted for each of these units of 3 wards. The target groups for the camps were residents of the wards above the age of 30 years. ASHAs registered individuals for the camps, going house to house and giving each target individual a registration slip giving his name, age and Unique health Identity number (UID) as entered from the Phase 1, with the advice to come on an empty stomach. The height, weight, fasting sugar, total cholesterol, blood pressure and physical examination, screening for refraction and cataract along with screening for anxiety and dependence on tobacco and alcohol was measured in individuals reporting for the camps. The blood was collected in labelled Vacutainer tubes giving the name, age and UID of the individual. The blood tests were done in the NABL accredited lab in the General Hospital, Ernakulam within 24 hours of collection.

The samples were transferred maintaining the cold chain. The details of blood tests were then entered on the individual data sheet using the UID, and was then passed on to the individual by ASHA's with the necessary advice. Educational material (CDs) regarding what constitutes a healthy diet and proper eating, benefits of exercise, benefits of yoga and meditation in health and other issues related to tobacco and alcohol use were screened during the course of each camp.

An individual was labelled diabetic if he or she had a fasting blood glucose value of $>110 \mathrm{mg} / \mathrm{dl}$. An individual was labelled hypertensive if he or she had a Systolic Blood pressure $>140 \mathrm{mmHg}$ or a diastolic blood pressure $>90 \mathrm{mmHg}$ or both in a single measurement. An individual was labelled hypercholesterolemic given a total cholesterol level of $>200 \mathrm{mg} / \mathrm{dl}$.

The sugars were estimated by the Glucose oxidase peroxidase (GOD-POD) method and blood pressure was measured on calibrated mercury sphygmomanometers. Total cholesterol was estimated by the CHOD-PAP (Cholesterol oxidase - peroxidase - 4 - phenol- 
aminoantipyrine) method on a fully automated analyser (Coralyser Tulip 200).

\section{Validation}

Validation of data entry was by way of telephoning each 25 th household to verify entry of details. In case contact was not established (which was rare), the next house on the list was contacted. Validation was also done by way of the medical camps done in the second phase in which the anthropometric measurements were repeated along with questions on lifestyle and disease and blood tests for sugar, cholesterol and measurement of the blood pressure. The weighing scales and measuring tape were calibrated before and after use.

The study was approved by the Institutional Ethics Committee of the MAGJ Hospital Mookkannoor (Refn: IEC-MAGJ-2011-CARD-57, Dated- 21/11/ 2011).

\section{Statistical analysis}

Chi-square test was used to find the association of risk factor with CAD. Forward conditional (multiple) binary logistic regression analysis was used to estimate the Odds of risk factors. Estimated risk (adjusted) expressed as OR with $95 \%$ confidence limit. The analysis was performed using IBM SPSS Statistics for Windows, Version 20.0. Armonk, NY: IBM Corp.

\section{RESULTS}

\section{Phase I}

Results as gathered by ASHAs through the oral questionnaire from 114,064 individuals in the house to house survey of all individuals in the 5 panchayats. The prevalence of non-communicable diseases was high (Table1). About $1 \%$ of the population reported being on treatment for coronary artery disease (CAD). The prevalence of cancer was $809 / 100,000$ individuals as per the survey. Behavioural risk factors were significant and almost exclusively limited to males. Close to $20 \%$ of the population were overweight or obese. Cerebrovascular accidents were only a fourth as common as CAD ${ }^{16-18}$ It was seen that the conventional risk factors including diabetes, hypertension, dyslipidemia, cancers, Chronic Obstructive Pulmonary diseases (COPD) were more prevalent in individuals with behavioural risk factors like tobacco and alcohol use. About $10 \%$ of the general population had a surgery done which could range from a cataract surgery to a CABG (coronary artery bypass graft), the only surgery being excluded from entry being PPS (peri-partum sterilisation procedures). The commoner surgeries were Cesarean sections, cataract surgeries, hysterectomies etc. The proportion of individuals having undergone a surgical procedure was higher among those with behavioural risk factors (Table 2).

Table 1: Prevalence of CVD, NCD and sociobehavioural factors contributing to disease in the total population surveyed and the target population.

\begin{tabular}{|l|l|l|}
\hline Disease condition & $\begin{array}{l}\text { General } \\
\text { population } \\
(\mathbf{n = 1 1 4 , 0 6 4 )})\end{array}$ & $\begin{array}{l}\text { Age } \mathbf{3 0} \\
\text { years (target } \\
\text { population } \\
\mathbf{n = 6 3 , 0 5 1 )}\end{array}$ \\
\hline Diabetes mellitus & $6.2 \%$ & $11 \%$ \\
\hline Hypertension & $8.7 \%$ & $15.6 \%$ \\
\hline Dyslipidemia & $3.9 \%$ & $6.9 \%$ \\
\hline $\begin{array}{l}\text { Coronary artery } \\
\text { disease }\end{array}$ & $1 \%$ & - \\
\hline $\begin{array}{l}\text { Cerebro-vascular } \\
\text { accidents (CVA) }\end{array}$ & $0.25 \%$ & - \\
\hline $\begin{array}{l}\text { Chronic respiratory } \\
\text { diseases }\end{array}$ & $4.5 \%$ & - \\
\hline Cancer & $0.8 \%$ & \\
\hline Tobacco use & $11.2 \%$ & $17.8 \%$ \\
\hline Alcohol use & $15.5 \%$ & $24.5 \%$ \\
\hline BMI $>\mathbf{2 5}$ & $19.1 \%$ & - \\
\hline
\end{tabular}

The prevalence of malignancies in the general population was $0.81 \%$ (Table 3 ). The commoner malignancies were those of the gastro-intestinal tract and liver, Breast, female genitalia, oro-pharynx, hematological and marrow and the lungs. Malignancies were more common in males $531(57.5 \%)$ than in females $392(42.5 \%)$.

\section{Phase II}

The blood sugar, total cholesterol and blood pressure was measured in close to 8000 individuals by way of camps. $48 \%$ of diabetics, $37 \%$ of hypertensives and $85 \%$ of dyslipidemics were unaware of their disease status. More females were unaware of their diabetic and lipid status, whereas more males were unaware of their hypertensive status (Table 5). About $25 \%$ of diabetics had proper sugar control, $40 \%$ of hypertensives had proper blood pressure control, while only $6 \%$ of dyslipidemics had normal values.

Table 2: Association between behavioural habits, NCD and surgeries.

\begin{tabular}{|lllllll|}
\hline Behavioural habits & Diabetes $(\%)$ & $\begin{array}{l}\text { Hypertension } \\
(\%)\end{array}$ & $\begin{array}{l}\text { Dyslipidemia } \\
(\%)\end{array}$ & $\begin{array}{l}\text { Cancer } \\
(\%)\end{array}$ & $\begin{array}{l}\text { COPD } \\
(\%)\end{array}$ & $\begin{array}{l}\text { Surgeries } \\
(\%)\end{array}$ \\
\hline $\begin{array}{l}\text { Tobacco use } \\
6429(19.8)\end{array}$ & $772(12)$ & $1057(16.4)$ & $423(6.6)$ & $116(1.8)$ & $792(12.3)$ & $931(14.5)$ \\
\hline $\begin{array}{l}\text { Alcohol use }- \\
9071(28)\end{array}$ & $1259(13.9)$ & $1684(18.6)$ & $795(8.8)$ & $188(2)$ & $985(10.9)$ & $1302(14.4)$ \\
\hline
\end{tabular}

Males - 59,114, males $>30$ years - 32,377. 
Table 3: The breakup of malignancy $(n=923)$.

\begin{tabular}{|ll|}
\hline Malignancy & $\mathbf{N}(\%)$ \\
\hline $\begin{array}{l}\text { Hematological and bone marrow } \\
\text { related }\end{array}$ & $72(7.8)$ \\
\hline Gastro-intestinal & $243(26.3)$ \\
\hline Oro-pharyngeal & $149(16.1)$ \\
\hline CNS & $39(4.2)$ \\
\hline Lungs & $58(6.3)$ \\
\hline Breast & $119(12.9)$ \\
\hline Gynaecological & $54(5.8)$ \\
\hline Liver and pancreas & $38(4.1)$ \\
\hline Uro-genital & $19(2.0)$ \\
\hline Soft tissue including thyroid & $45(4.9)$ \\
\hline Unknown primary & $97(10.5)$ \\
\hline
\end{tabular}

\section{Tobacco use score}

The prevalence of tobacco use was $11.2 \%$.Tobacco abuse was also scored through the Fagerstrom's test for nicotine dependence. 3533 individuals who consented for the questionnaire reported no tobacco use. 36(1\%) individuals had a score of 5 signifying moderate tobacco dependence. Of the 36 individuals with moderate tobacco dependence, 28 also reported regular use of alcohol.

The rates of tobacco use and especially alcohol use have been underreported as the participants were not willing to disclose the use of tobacco and alcohol (Table 6). These figures we feel are gross underestimates as a significant number of individuals who came for the camp were unwilling to open up regarding their social habits.

Table 4: Phase II of the study.

\begin{tabular}{|lll|}
\hline \multirow{2}{*}{$\begin{array}{l}\text { Dests } \\
\text { mellitus }\end{array}$} & $\begin{array}{l}\text { Number (tested) } \\
\text { males }\end{array}$ & $\begin{array}{l}\text { Prevalence } \\
(\%)\end{array}$ \\
\cline { 2 - 3 } & Males 2738 (592) & 20.20 \\
\cline { 2 - 3 } Hypertension & Females 4883 (950) & 19.4 \\
\cline { 2 - 3 } & 7238 & 20.30 \\
\cline { 2 - 3 } & Males 2583 (624) & 24.10 \\
\hline \multirow{3}{*}{ Dyslipidemia } & Females 4665 (848) & 18.10 \\
\cline { 2 - 3 } & Males 2750 (1561) & 56.8 \\
\cline { 2 - 3 } & Females 4592 (3068) & 66.8 \\
\hline
\end{tabular}

Table 5. Awareness, treatment and adequacy of control of diabetes, hypertension and dyslipidemia.

\begin{tabular}{|c|c|c|c|c|c|}
\hline Risk factors & Gender & $\begin{array}{l}\text { Aware and } \\
\text { treated } \\
\mathbf{N}(\%)\end{array}$ & $\begin{array}{l}\text { Aware and } \\
\text { untreated } \\
\mathbf{N}(\%)\end{array}$ & $\begin{array}{l}\text { Unaware and } \\
\text { untreated } \\
\mathbf{N}(\%)\end{array}$ & $P$ value \\
\hline \multirow{3}{*}{ Diabetic $(n=2100)$} & Female & $310(24.0)$ & $339(26.2)$ & $645(49.8)$ & \multirow{3}{*}{0.029} \\
\hline & Male & $199(24.7)$ & $249(30.9)$ & $358(44.4)$ & \\
\hline & Total & $509(24.2)$ & $588(28.0)$ & $1003(47.8)$ & \\
\hline \multirow{3}{*}{ Hypertension $(n=2394)$} & Female & $629(43.0)$ & $336(23.0)$ & $497(34.0)$ & \multirow{3}{*}{0.001} \\
\hline & Male & $334(35.8)$ & $219(23.5)$ & $379(40.7)$ & \\
\hline & Total & $963(40.2)$ & $555(23.2)$ & 876 (36.6) & \\
\hline \multirow{3}{*}{ Cholesterol $(n=4665)$} & Female & $156(5.0)$ & $280(9.0)$ & $2658(85.9)$ & \multirow{3}{*}{$<0.001$} \\
\hline & Male & $120(7.6)$ & $181(11.5)$ & $1270(80.8)$ & \\
\hline & Total & $276(5.9)$ & $461(9.9)$ & $3928(84.2)$ & \\
\hline
\end{tabular}

Table 6: Males $(n=4048)$ were screened by way of an Alcohol scoring for alcohol abuse (AUDIT score).

\begin{tabular}{|lll|}
\hline Alcohol score & $\begin{array}{l}\text { Dependence } \\
\mathbf{0}\end{array}$ & $\begin{array}{l}\text { Number of } \\
\text { individuals }\end{array}$ \\
\hline $\mathbf{1 - 7}$ & $\begin{array}{l}\text { No use } \\
\text { harmful use }\end{array}$ & 3306 \\
\hline $\mathbf{8 - 1 5}$ & Harmful, hazardous use & 138 \\
\hline $\mathbf{1 6 - 1 9}$ & $\begin{array}{l}\text { Mild alcohol } \\
\text { dependence }\end{array}$ & 22 \\
\hline $\mathbf{2 0 - 4 0}$ & $\begin{array}{l}\text { Severe alcohol } \\
\text { dependence }\end{array}$ & 36 \\
\hline
\end{tabular}

The score used was the AUDIT- Alcohol Use Disorders Identification Test.

\section{DISCUSSION}

The primary goal of the "ENDIRA" study was to look at the prevalence of non-communicable diseases in a population of $>100,000$ individuals. The self-reported prevalence of DM, HT, dyslipidaemia in a population of 1 lakh was $6.2 \%, 8.7 \%, 1 \%, 3.9 \%$ respectively which increased to $11 \%, 15 \%$ and $19 \%$ in adults $>30$ years of age. The fact that we did blood tests by way of camps in the very near vicinity of their homes encouraged more number of individuals to actually get their blood tests done, in the target group of individuals $>30$ years $(n=63051)$. In this way we were able to partially risk stratify a number of individuals who had never undergone an assessment before. 
Authors had given each individual in the 5 panchayats a health-UID, something akin to an Aadhaar number. Details regarding each individual are available on his/her own webpage accessible to only him/her or the administrator, which could be accessed on the website www.endira.in. The health details gathered have been transferred to the respective PHCs (Primary health centres) where follow up is maintained.

In the first phase the NCDs in the general population was quantified through an oral questionnaire. As a follow up, in a select target group of individuals above the age of 30 blood tests, physical parameters and screening for tobacco and alcohol dependence and wellness scoring was done by way of camps. In parallel, educational material on the benefits of yoga and meditation, what comprises a healthy diet and good eating habits along with the ills related to tobacco and alcohol use were screened during all the camps.

The long term goal is to convert it into a cohort. The ENDIRA group would be re-doing the survey again in the study population. The same UID given to individuals would be used for data entry, this time, on a tablet PC. This would help us arrive at the incidence of NCDs in the study population. We would also be looking at compliance to drugs and adverse events in individuals with documented CVD.

We are also looking at follow up by way of mobile phones- a M-health program in the cohort a small pilot of which has already been done. ${ }^{18,19}$

The drawbacks of the study were in phase 1, self reporting has been used as a measure of the noncommunicable disease conditions. The number of CVA's would not include a large number of Transient Ischemic attacks (TIA's) especially if the individual concerned has not been continuing medicines. The list of cancers given may not be a true representation as throat surgeries may have been reported as thyroid and vice-versa. Data on the educational status and income has not been collected, which would have been invaluable. Dietary pattern included, was on use of red meats and the type of oil used while information on including fruits and vegetable intake was not included.

In the Phase 2 only about 8000 individuals of the 64,000 in the target group of $>30$ years could be reached out to. This was about $12.5 \%$ only, while the target we had set was $20 \%$, keeping in mind that we could only accommodate about 800 individuals in a single camp. There was an inherent bias in individuals reporting for the camp as individuals with proven disease and those who are more aware are the ones more likely to report for camps. More number of females reported for the camps as compared to males. We have not been able to measure as yet the impact of life-style modification that we think we have been able to bring about. The success of life- style changes would take a few years to have an impact and our next step is in that direction.

Our community level data on the prevalence and awareness of NCDs, parallel the findings of previous studies in South Kerala. ${ }^{6}$ On the socio-behavioural aspects as per the study use of tobacco products was identified in $28 \%$ and alcohol use in $15.4 \%$ of those studied while it was $17.8 \%$ and $24.5 \%$ respectively in our study (ENDIRA). The prevalence of hypertension $(28.8 \%)$, diabetes $(14.8 \%)$ and dyslipidemia $(54.1 \%)$ as compared to HTN (20.3\%), diabetes (20.2\%) and dyslipidemia (60.1\%) in the ENDIRA study. ${ }^{6,20}$ The awareness of NCDs in the previous study was $36.9 \%$ for hypertension and $72.2 \%$ for diabetes which contrasts with our findings of $47.8 \%$ of individuals with high sugars, $36.6 \%$ with high blood pressure and $84.2 \%$ of individuals with high cholesterol being unaware of disease.

The ENDIRA group has worked in close coordination with the District health authorities allowing for continuity of care at the local primary health centre (PHC) itself. A concerted effort is sure to bring about a life style change towards good health in families especially when women and children are targeted, this specially so in families with NCD or CVD.

\section{CONCLUSION}

The study has suggested low levels of awareness of NCDs in the study population with poor control of disease in those on treatment. The prevalence of modifiable risk factors like tobacco and alcohol use continues to be high in spite of sustained efforts by NGOs and the government to curb the same. CVD, cancer and COPD was much more common in individuals with behavioural risk factors and Obesity suggesting efforts to curb the same would greatly help disease progression and there should be an all out effort on the part of both the government and health care professionals to create more awareness and take steps to tackle alcohol and tobacco use. Awareness of the disease condition is a first step to its care. The study should also serve as an eye opener to health officials on the degree of unawareness and there should be a mass screening on a regular basis through the public health system.

Funding: No funding sources Conflict of interest: None declared

Ethical approval: The study was approved by the Institutional Ethics Committee

\section{REFERENCES}

1. Whiting DR. IDF Diabetes atlas: global estimates of the prevalence of diabetes for 2011 and 2030, diabetes research and clinical practice. Available at: http://www.sciencedirect.com/science/article/pii/S01 68822711005912). Accessed on 11 February 2020. 
2. Lago RM, Singh PP, Nesto RW. Diabetes and hypertension. Nature Clin Practice Endocrinol Metab. 2007;3(10):667.

3. Goyal A. The burden of cardiovascular disease in the Indian subcontinent, 2006. Available at: www. icmr. nic. in/ ijmr/ 2006/ september/ 0903. pdf. Accessed on 11 February 2020.

4. Economic implications of chronic diseases in India, South Asia network for chronic diseases. Available at https://catalog.ihsn.org/index.php/citations/52429. Accessed on 11 February 2020.

5. CADI Research Foundation: Coronary artery disease in Asian Indians. Available at: https://cadiresearch.org/. Accessed on 18 February 2020.

6. Thankappan KR, Shah B. Risk factor profile for chronic non-communicable diseases: results of a community-based study in Kerala, India. Indian J Med Res. 2010;131:53-63.

7. Krishnan MN, Zachariah G. Prevalence of coronary artery disease and its risk factors in Kerala, South India: a community-based cross-sectional study. BMC Cardiovasc Disord. 2016;16:12.

8. Gupta R, Gupta S, Deedwania P. Regional variations in cardiovascular risk factors in India: India heart watch. World J Cardiol. 2012;4(4):11220.

9. Mohan V, Seedat YK, Pradeepa R. The rising burden of diabetes and hypertension in Southeast Asian and African regions: need for effective strategies for prevention and control in primary health care settings. Int J Hyperten. 2013;409083:114.

10. Patel A, Mohanan PP, Prabhakar D. Pre-hospital acute coronary syndrome care in Kerala, India: a qualitative analysis. Indian Heart J. 2017;69(1):93100.

11. Krishnan MN. Coronary heart disease and risk factors in India - on the brink of an epidemic? Indian Heart J. 2012;64(4):364-7.

12. Radwan I. India private health services for the poor. Availale at: http:// documents. worldbank.org /curated / en/ $843261468033291849 /$ India-privatehealth-services-for-the-poor. Accessed on 10 January 2020.

13. Pauly MV, Zweifel P, Scheffler RM, Preker AS, Bassett M. Private health insurance in developing countries. Health Aff. 2006;25:369-79.
14. National health mission: prevention and control of non-communicable diseases programmes, Ministry of Health and Family Welfare. Available at: http://www.mohfw.nic.in/index 1.php?lang=1\&level $=2 \&$ sublinkid $=659 \&$ lid $=651$. Accessed on 10 January 2020.

15. Huffman M, Mohanan PP, Prabhakar D. Effect of a quality improvement intervention on clinical outcomes in patients in India with acute myocardial infarction, The ACS-QUIK randomised controlled trial. JAMA. 2018;319(6):567-78.

16. Menon J, Jacob J, Atacheril TV. Surveillance of non-communicable diseases by community health workers in Kerala the epidemiology of noncommunicable diseases in rural areas (ENDIRA) study. Glob Heart. 2014;9(4):409-17.

17. Menon J, Vijayakumar N, Joseph J. Below the poverty line and non-communicable diseases in Kerala: the epidemiology of non communicable diseases in rural areas (ENDIRA) study. Int J Cardiol. 2015;187:519-24.

18. Smith R, Menon J, Banerjee A. Potential for the use of mhealth in the management of cardiovascular disease in Kerala: a qualitative study: BMJ Open. 2015;5:009367.

19. Feinberg L, Menon J, Banerjee A. Potential for mobile health (mHealth) prevention of cardiovascular diseases in Kerala: a populationbased survey. Indian Heart J. 2017;69(2):182-99.

20. Danaei G, Finucane MM, Lin JK. National, regional, and global trends in systolic blood pressure since 1980: systematic analysis of health examination surveys and epidemiological studies with 786 country-years and 54 million participants. Lancet. 2011;377(9765):568-77.

Cite this article as: Menon JC, Sreedevi A, Beena KV, Rajesh T, Attacheril TV, Jaisoorya TS. Noncommunicable diseases awareness and control in a rural population in an epidemiologically advanced stage of transition (Kerala): results of the epidemiology of non-communicable diseases in rural areas study. Int J Community Med Public Health 2020;7:2628-34. 